\title{
Genomics in non-adenoid cystic group of salivary gland cancers: one or more druggable entities?
}

\author{
Stefano Cavalieri, Francesca Platini, Cristiana Bergamini, Carlo Resteghini, \\ Donata Galbiati, Paolo Bossi, Federica Perrone, Elena Tamborini, Pasquale \\ Quattrone, Lisa Licitra, Laura Deborah Locati \& Salvatore Alfieri
}

To cite this article: Stefano Cavalieri, Francesca Platini, Cristiana Bergamini, Carlo Resteghini, Donata Galbiati, Paolo Bossi, Federica Perrone, Elena Tamborini, Pasquale Quattrone, Lisa Licitra, Laura Deborah Locati \& Salvatore Alfieri (2019): Genomics in non-adenoid cystic group of salivary gland cancers: one or more druggable entities?, Expert Opinion on Investigational Drugs, DOI: $\underline{10.1080 / 13543784.2019 .1598376}$

To link to this article: https://doi.org/10.1080/13543784.2019.1598376

Accepted author version posted online: 22 Mar 2019.

Submit your article to this journal $\pi$

View Crossmark data $₫$ 
Publisher: Taylor \& Francis

Journal: Expert Opinion on Investigational Drugs

DOI: $10.1080 / 13543784.2019 .1598376$

Genomics in non-adenoid cystic group of salivary gland cancers: one or more druggable entities?

Stefano Cavalieri ${ }^{1}$, Francesca Platini ${ }^{1}$, Cristiana Bergamini ${ }^{1}$, Carlo Resteghini ${ }^{1}$, Donata Galbiati ${ }^{1}$, Paolo Bossi ${ }^{1}$, Federica

Perrone $^{2}$, Elena Tamborini2 ${ }^{2}$ Pasquale Quattrone ${ }^{2}$, Lisa Licitra ${ }^{1}{ }^{3}$, Laura Deborah Locati1 and Salvatore Alfieri ${ }^{1}$

${ }^{1}$ Head and Neck Cancer Medical Oncology 3 Unit. Fondazione IRCCS Istituto Nazionale dei Tumori di Milano. Via

Venezian 1, 20133 Milan, Italy.

2Pathology Department. Fondazione IRCCS Istituto Nazionale dei Tumori di Milano. Via Venezian 1, 20133 Milan, Italy.

3University of Milan. Via Festa del Perdono 7, 20122 Milan, Italy

\section{Corresponding Author:}

Salvatore Alfieri, Head and Neck Cancer Medical Oncology 3 Unit. Fondazione IRCCS Istituto Nazionale dei Tumori di Milano. Via Venezian 1, 20133 Milan, Italy.

Email: salvatore.alfieri@istitutotumori.mi.it

Phone: +39-0223902765/+39-0223902150

Keywords: salivary gland cancer, genomics, druggable, targeted therapy, salivary duct carcinoma, MASCC 


\begin{abstract}
Introduction: Salivary gland cancers (SGCs) are a rare and heterogeneous group of malignant tumors arising from either major or minor salivary glands. Among SGCs patients, adenoid cystic carcinoma (ACC) is the most frequent histotype and its genetic aberrations are well known even though they are generally uncommon. Non-ACC subtypes are rarer and more heterogeneous than ACC from a histological and genomic point of view. In non-ACC, some altered molecular pathways [e.g. BRAF or RET mutations, Androgen Receptor (AR), etc.] are potentially targetable with specific drugs.
\end{abstract}

Areas covered: A literature search was performed to summarize the main druggable genomic aberrations involving nonACC SGCs. An overview of the genomics of non-ACC salivary gland malignancies is discussed. We describe the pattern of potentially targetable genomic alterations in non-ACC salivary gland malignancies according to their frequency rather than to the single non-ACC histotype

Expert Opinion/Commentary: The genetic profiling through in-depth molecular analyses [e.g. Next-generation sequencing (NGS)] is advised in all patients affected by recurrent and/or metastatic non-ACC SGCs to find any potentially druggable target. Some histotypes may carry driving mutations (e.g. salivary duct carcinoma and AR expression) that must be investigated and defined. For the rare cancers, access to a referral center is recommended to optimize the management of these patients. 


\section{Article Highlights}

1. Non-adenoid cystic carcinomas (non-ACCs) are rarer and more heterogeneous than ACC as subtypes of salivary gland malignancies

2. Non-ACC have higher rates of genomic alterations than ACC

3. Some genomic alterations recurring in non-ACC are potentially druggable

4. In-depth molecular analyses are advised in patients with advanced non-ACC

5. Access to referral centers is recommended to optimize the management of non-ACC patients 


\section{Introduction}

Salivary gland cancers are a rare and heterogeneous group of malignant tumors arising from either major or minor salivary glands [1].

Among metastatic salivary gland cancer patients, the most frequent histotype is adenoid cystic carcinoma (ACC) [2]. This is even the most widely studied histotype from a genomic point of view [3] [4], while non-ACC salivary gland cancers (Table 1) have been often fragmentarily analyzed [5]. The genomic landscape of salivary gland cancers was already explored in 2015 [6] [7], and a comprehensive overview of genomic profiling of more than 600 salivary gland cancers was recently published [8]. For example, we know that high grade salivary gland malignancies [e.g. adenocarcinoma not otherwise specified and salivary duct carcinoma] have a higher number of genetic alterations (4.1 and 3.6 genomic alterations per tumor, respectively) than low grade ones (e.g. polymorphous adenocarcinoma and acinic cell carcinoma, with 1.6 and 2.8 genomic alterations per tumor, respectively).

Keeping in mind that salivary gland cancers are commonly characterized by a lower rate of tumor DNA mutations if compared to other malignancies [9], some molecular alterations may be of particular interest because druggable.

In this review, we describe the pattern of potentially targetable genomic alterations in non-ACC salivary gland malignancies according to their frequency rather than to the single non-ACC histotype (Table 2). In this context, TP53 is one of the most mutated genes and it is usually related to differentiation grade [8] and aggressiveness of salivary gland carcinomas [10], but it will not be discussed as still not druggable.

\section{Genomic alterations}

\subsection{Hormone receptors}

Within a cohort of 139 cases of salivary gland cancers, $13 \%$ of tumors (all cases of salivary duct carcinoma or adenocarcinoma not otherwise specified - NOS) expressed androgen receptor (AR) [11]. Through IHC, AR expression can be identified at nuclear level in almost all salivary duct carcinoma cells [12]. Indeed, AR expression is the hallmark of salivary duct carcinoma (75-99\%) and it was reported in $21 \%$ of adenocarcinoma NOS (Table 2).

AR expression is a phenomenon related to apocrine differentiation which is the case in SDC, unrelated to gender. However, the higher prevalence of male gender ( $71 \%$ of the cases) and the advanced age at the time of diagnosis (median age of 66 years) corroborate the role of hormones as pathogenic factor for these cancers [13]. Gene amplification seems to be not involved even if almost $40 \%$ of SDC may have an extra copy of chromosome $X$, which 
includes the AR gene [14] [15]. Differently from prostate cancer, amplification or somatic mutations leading to altered proteins of AR have not been found in salivary gland carcinomas [16].

This finding led to the development of androgen deprivation therapy (ADT) in recurrent and/or metastatic AR-positive salivary duct carcinomas and adenocarcinoma NOS. In fact, ADT demonstrated a significant activity first in retrospective analyses [overall response rate (ORR) ranging between 50\% and 64.7\% [17] ] and recently in a prospective series (ORR $=41.7 \%$ ) [18]) with a durable clinical benefit (11-months as duration of response for patients with partial response or stable disease [19]). As observed in prostate cancer, AR-positive salivary gland malignancies resistant to conventional ADT might benefit from novel anti-AR agents, such as abiraterone acetate [20] and enzalutamide [21]. Two prospective trials with abiraterone and enzalutamide are currently ongoing in AR-positive SGCs patients progressed on a first ADTline. Genetic variants of $A R$, notably AR-v7, have been reported as one of the mechanism of ADT resistance in prostate cancer [16]. Interestingly, circulating tumor cells expressing AR-v7 have been recently described in a patient with ARpositive salivary duct carcinoma not responding to second-line hormone therapy (abiraterone) [22], supporting a similar role for AR-v7 in salivary gland cancers and paving the way for a potential role for liquid biopsy even in the management of these patients.

Despite the similar morphological behavior between salivary duct and breast cancers (notably SDC and breast apocrine carcinoma [23]), progesterone (PgR) and estrogen receptors (ER) are usually not expressed in salivary duct carcinomas [11], hence they should not be routinely investigated in these patients.

\subsection{EGFR family}

The family of epidermal growth factor receptor (EGFR) include a variety of transmembrane proteins whose main ligand is epidermal growth factor. In clinical practice, anti-EGFR compounds including monoclonal antibodies (e.g. cetuximab) [24] and tyrosine kinase inhibitors (e.g. gefitinib) [25] failed to demonstrate any clinical activity in salivary gland carcinomas. This lack of activity might be owed to the rarity of EGFR mutations (e.g. $5 \%$ of salivary duct carcinomas) or its infrequent overexpression in these rare tumors (63\% of non-ACC, especially high-grade mucoepidermoid carcinomas and adenocarcinoma NOS) [8] [26].

A median frequency of $13 \%$ of human epidermal growth factor receptor type 2, (HER-2/ERB-B2) genomic alterations, notably amplifications, was reported in high grade salivary gland cancers [8]: $32 \%$ [8] to $73 \%$ [27] in salivary duct carcinoma, $15-17 \%$ in adenocarcinoma NOS and $13 \%$ in mucoepidermoid carcinoma [8]. In general, HER-2 genomic alterations are constantly absent in low grade and indolent cancers [8].

HER-2 overexpression has been observed in some non-ACC (e.g. 11\% mucoepidermoid carcinoma , <1\% acinic cell carcinoma, $21 \%$ salivary duct carcinoma). HER-2 amplification is much rarer (e.g. $1 \%$ mucoepidermoid carcinoma ; $2 \%$ myoepithelial carcinoma), being more frequently restricted to salivary duct carcinoma $(21 \%)$. Indeed, only $18 \%$ of SGCs with 2+ or 3+ at HER-2 immunohistochemistry (IHC) had a HER-2 amplification in fluorescence in-situ hybridization (FISH) [28]. Furthermore, HER-2 amplification and an increased gene copy number of EGFR were found to be 
associated with high grade salivary gland cancers, loss of PTEN and poor prognosis [29] [30]. This is interestingly similar to what is found in lacrimal gland ductal carcinoma(e.g. salivary duct carcinoma) [31].

Anti-HER-2 agents have been explored in recurrent or metastatic salivary gland cancers. Notably, a prolonged response was observed in high-grade HER-2 positive (3+ at IHC) mucoepidermoid carcinoma treated with trastuzumab [32] [33]. Lapatinib did not show any clinical responses in 17 non-ACC patients treated within a phase II study [34]. Double HER-2 blockade with trastuzumab and pertuzumab in HER-2 amplified salivary gland malignancies is under evaluation within clinical trials [35]. Cases of HER-2 amplified SGCs patients responding to trastuzumab-emtansine were reported as well [36]. Since HER-2 amplifications can be observed in a third of cases, all salivary duct carcinoma and adenocarcinoma NOS patients ought to undergo HER-2 screening. These subjects might benefit from anti-HER-2 therapies. Indeed, a recent Japanese phase II study demonstrated safety and activity of trastuzumab and docetaxel in HER-2 positive salivary duct carcinoma [37].

Moreover, a combined AR and HER-2 expression was seen in 56\% (14/25) of salivary duct carcinoma and in 19\% (3/16) of poorly differentiated salivary gland cancers [38]. This might be an epiphenomenon reflecting both the hormoneinduced cancerogenesis and HER-2-driven tumor aggressiveness. . The identification by IHC of AR and HER-2 status should be explored in all cases of salivary duct carcinoma and adenocarcinoma NOS.

\subsection{PI3K pathway}

Phosphatidylinositol-3-kinase (PI3K) pathway includes many actors, potentially harboring genomic alterations: PI3K catalytic subunit alpha (PIK3CA) mutations (overall, 19\% in non-ACC [7]: $27 \%$ salivary duct carcinoma, 20-24\% adenocarcinoma NOS, 20\% mucoepidermoid carcinoma, 15\% myoepithelial carcinoma [8]), RICTOR (mTOR subunit) mutations (10\% myoepithelial carcinoma), and PTEN (a tumor suppressor gene coding for an antagonist of the PI3K pathway) mutations ( $20 \%$ polymorphous adenocarcinoma, $17 \%$ salivary duct carcinoma, $10 \%$ acinic cell carcinoma, $8 \%$ adenocarcinoma NOS, especially in ADT-resistant ones [20]).

In addition to these mutations, genomic alterations of PI3K pathway (PIK3CA, PIK3R1, PTEN or AKT1/3 aberrations) were observed in salivary gland malignancies (53.6\% salivary duct carcinoma [39] [40]; 40\% mucoepidermoid carcinoma, $33 \%$ myoepithelial carcinoma, $14 \%$ acinic cell carcinoma [7]). In a large cohort of mucoepidermoid carcinoma patients $(n=48)$, activation of PI3K/mTOR pathway varies according to tumor differentiation grade: $52 \%$ in high grade vs $4.3 \%$ in low and intermediate grade tumors [39]. However, in mucoepidermoid carcinoma, no correlation between phosphorilated kinases and tumor differentiation grade was observed [41].

Since many agents targeting PI3K/mTOR/AKT (e.g. everolimus, temsirolimus, idelalisib etc.) are currently available in clinical practice, the role of this pathway deserves more attention, especially in salivary duct carcinoma, adenocarcinoma NOS and in mucoepidermoid carcinoma. 


\subsection{TRK}

Tropomyosin receptor kinases (TRK) are a group of transmembrane receptors binding nerve growth factor (NGF). In 2010 , Skalova et al. first described a subset of salivary gland cancers, formerly diagnosed as acinic cell carcinoma or cystadenocarcinoma, resembling secretory carcinoma of the breast and bearing $t(12 ; 15)$ (p13;q25) ETV6-NTRK3 translocation [42]. Thereafter, this particular tumor type was recognized as mammary analogue secretory carcinoma (MASC) becoming a specific pathologic entity in the landscape of non-ACC group included in the WHO classification for the first time in 2017. Besides, it is worth noticing that not all secretory carcinomas have NTRK3 as fusion partner, a subset has an ETV6-RET fusion which is shared only with a rare subtype of sinonasal carcinoma [43] [44] [45]. MASC is usually indolent and patients are effectively cured by loco-regional treatments. Thus, systemic therapies are not indicated except for sporadic cases of metastatic disease. Specific drugs, as entrectinib [46] and larotrectinib [47], exert a pan-TRK blockade, including the product of ETV6-NTRK3 translocation. TRK inhibitors showed activity in cases of recurrent or metastatic mammary analogue secretory carcinoma [48]. An impressive activity of entrectinib within three phase I/II basket trials (ALKA, STARTRK-1, STARTRK-2) was recently reported across tumors harboring NTRK rearrangement [49]. Due to these promising results, all patients with advanced ETV6-NTRK3 translocated mammary analogue secretory carcinomas requiring systemic therapies deserve to be treated with TRK inhibitors.

\subsection{Cyclins/Cyclins dependent kinases (CDK)}

Cyclins are proteins involved in cell cycle control. Specific cyclin-dependent kinase inhibitors (CDK-I) have a major role in cancer therapy. In detail, in ER-positive breast cancer, CDK-Is (palbociclib and abemaciclib) associated with hormone therapy significantly improved outcome in the recurrent/metastatic setting [50] [51], also in terms of overall survival [52]. Genomic alterations of the cyclins/cyclin-dependent kinase (CDK) pathway (CCND1, CDK4/6 or CDKN2A/B aberrations) were found in approximately one third of non-ACC, particularly in acinic cell carcinoma $(71 \%)$, undifferentiated carcinoma (67\%), adenocarcinoma NOS (35\%), myoepithelial carcinoma (33\%) and mucoepidermoid carcinoma (20\%) [7]. With regards to prevalence of CDKN2A and CDKN2B mutations in salivary gland malignancies, mucoepidermoid carcinoma was the most frequently mutated histotype, where these two mutations were respectively observed in $45 \%$ and $32 \%$ of cases [8]. Therefore, the role of CDK-Is should be investigated for treatment in those mutated patients with advanced disease.

\subsection{FGFR}

Fibroblast growth factor receptor (FGFR) and its ligand (FGF) are involved in cancer development and disease progression. Specific FGFR inhibitors are currently under evaluation in medical oncology [53]. Among non-ACC, polymorphous adenocarcinoma (20\%), carcinoma ex pleomorphic adenoma (9\%) and mucoepidermoid carcinoma (7\%) resulted as the most frequently FGFR-mutated histotypes [8]. Genomic aberrations of FGFR1 and FGFR3, together with TP53 product regulators (e.g. MDM2 and MDM4), were associated with dismal prognosis in patients with salivary gland 
cancers [54]. Rearrangements involving FGFR gene, notably gene fusions in myoepithelial carcinoma, will be described in a dedicated paragraph below [55].

Both non selective (e.g. nintedanib, lenvatinib, dovitinib, lucitanib) and selective drugs inhibiting FGFR/FGF axis are currently available or still under evaluation in medical oncology [53].

\subsection{BRCA}

Germ-line mutations of breast related cancer antigens 1 and 2 (BRCA1 and BRCA2) genes are associated with an increased risk of cancer development, especially breast and ovarian cancer. In the context of non-ACC, mucoepidermoid carcinoma was the unique type associated with somatic BRCA mutations (10.5\% of BRCA1/2 genomic alterations [39], $17 \%$ of BRCA2 mutations [8]). It is interesting to note as in this work [8] there were no data about BRCA germ-line mutations. Therefore, it is emphasizing that there is no association between germ-line BRCA mutations and salivary gland cancers. BRCA mutations lead to DNA misrepair and poly-ADP ribose polymerase inhibitors (PARPi) are drugs able to enhance the genetic instability and eventually to cell death. This leads to a potent anti-cancer activity known as synthetic lethality [56]. This genetic instability might be induced by conventional chemotherapies as well, in particular by platinum-based treatments. Indeed, BRCA pathway was found to be a major predictor of response to cisplatin in head and neck squamous cell cancer (HNSCC) patients treated with chemo-radiation [57]. Therefore, BRCA-mutated MEC might benefit from platinum-based chemotherapy with or without PARPi (e.g. olaparib, talazoparib, rucaparib, niraparib, veliparib etc.) in the recurrent/metastatic setting. Moreover, due to these biologic findings, cisplatin might have a role in the adjuvant setting, particularly in post-operative chemo-radiation for radically resectable BRCA-mutated mucoepidermoid carcinomas.

\subsection{RAS, RAF and MAP kinases}

Abnormalities in HRAS were observed in $16 \%$ of non-ACC [7], and in this subset they were frequently associated with PI3K pathway aberrations. BRAF mutations can be found in a minority of acinic cell carcinoma, salivary duct carcinoma and adenocarcinoma NOS (5\% each) [8]. In detail, BRAF V600E mutation was described in $7 \%$ of salivary gland malignant tumors [58], and MAP kinases (including NF1) were frequently altered in salivary duct carcinomas [16]. Since specific agents targeting RAS mutation variants (e.g. tipifarnib [59]), BRAF V600 mutations (e.g. vemurafenib, dabrafenib, encorafenib), and MAPK (e.g. cobimetinib, trametinib, binimetinib) are under evaluation or already available in the clinical practice, the identification of these mutations should be warranted in entire non-ACC population.

\subsection{Hedgehog}


Actors in sonic hedgehog signaling pathway are Hedgehog, its transmembrane receptor PTCH1, the transmembrane protein SMO and downstream protein kinases and GLI transcription factors [60]. This complex biologic system is frequently associated with cancer development [61], notably in basal cell carcinoma. This pathway was found to be involved in the development and maintenance of any grade mucoepidermoid carcinoma [62]. Moreover, PTCH1 mutations can be found in $10 \%$ of myoepithelial carcinoma [8]. Since a specific hedgehog inhibitor vismodegib is active and well tolerated in metastatic or unresectable basal cell carcinoma [63], the use of this drug or its analogues (e.g. sonidegib) could be taken into account in case of PTCH1 mutated MEC or myoepithelial carcinoma [64].

\subsection{MET}

MET gene codifies for the hepatocyte growth factor receptor (HGFR). The activation of this pathway is involved in some type of cancers, notably non-small cell lung cancer (NSCLC) [65] [66], and in Hepatocellular carcinoma (HCC) [67]. In a German cohort of 198 non-ACC patients (233 in total, 35 ACC), MET was deleted in only 9\% of cases [68]. In the majority of non-ACC (57\%), a physiological MET disomy was found. Approximately one third of cases had either a polysomy or an amplification of MET gene. The only two cases bearing MET amplification were one adenocarcinoma NOS and one myoepithelial carcinoma. The activity of several MET inhibitors (e.g. crizotinib, capmatinib, cabozantinib etc.) has been explored in NSCLC. However, due to rarity of MET amplification in salivary gland cancers and its general difficulty to be targeted, this test should be performed only in case of lack of all druggable alterations.

\subsection{Tumor mutational burden}

Genetic instability which is typical of some tumors, such as NSCLC in smokers or colorectal cancer with high microsatellite instability (MSI), is associated with generation of neo-antigens. These may elicit an immune response, which can also be enhanced by specific immune checkpoint inhibitors. In the past few years, immune-oncology (IO) agents, like anti-programmed death (PD)-1 or anti its ligand (PD-L1) and anti-CTLA4 (common T lymphocyte antigen 4) monoclonal antibodies, have been introduced in the clinical practice for several advanced tumors, such as melanoma, NSCLC, HNSCC, urothelial and renal cancers. The rate of tumor DNA mutations per megabase (mut/MB) is known as tumor mutational burden (TMB). Cancer cells with high TMB are strongly immunogenic. Indeed, higher responses to IO agents have been observed in tumors with higher TMB, when compared to the same cancers with lower TMB [69] [70].

In their comprehensive analysis of recurrent or metastatic SGCs, Ross et al. reported the frequency of TMB (defined as $>10$ mut/MB) in SGCs. An inverse relationship between TMB and tumor differentiation grade was observed in non-ACC. In particular, the histotype with the highest TMB was salivary duct carcinoma (14\%), followed by carcinoma ex pleomorphic adenoma (12\%), mucoepidermoid carcinoma and adenocarcinoma NOS (10\% each) [70]. This frequency is significantly lower than what can be observed in other malignancies: $32 \%$ of intermediate TMB (6-19 mut/MB) and 25\% of high TMB ( $\geq 20$ mut/MB) in a heterogeneous cohort of patients affected by different cancers (mostly melanoma and NSCLC) and treated with 10 agents [71]. 
With regards to TMB, many controversies have to be completely solved yet. In fact, even though TMB was theoretically associated with higher immunogenicity, the correlation between TMB and response to $\mathrm{IO}$ agents was not always strong [72]. Moreover, an univocal threshold value of genetic mutations, needed to define TMB as "high", has still far to go.

\subsection{PD-1 and PD-L1}

PD-1 and its ligands (PD-L1 and PD-L2) are molecular checkpoints involved in the regulation of the immune response. The activation of PD-1 induces apoptosis of antigen-specific T cells and regulatory T lymphocytes. Tumor cells can use these molecular checkpoints in order to escape the anti-tumor immune response [73]. In brief, this is the rationale to use anti-PD-1 or anti-PD-L1/PD-L2 agents in medical oncology. These molecules can be easily found by $\mathrm{HC}$ both on tumor cells or antigen presenting cells (PD-L1 and PD-L2) and on B and T lymphocytes (PD-1) [74]. The association between PD-1/PD-L1 expression and clinical response to 10 agents may depend on tumor type. The response to anti-CTLA4 (ipilimumab) plus anti-PD-1 (nivolumab) in melanoma was regardless of PD-L1 expression [75], whereas PD-L1 positive NSCLC patients had benefit from anti-PD-1 pembrolizumab significantly better than PD-L1 negative ones [76].

In a retrospective analysis of 219 salivary gland cancer cases, a higher PD-L1 expression was observed in high grade tumors rather than in low grade ones ( $90 \%$ vs $10 \%$ ). The most frequently PD-L1 positive (defined as $\geq 1 \%$ of tumor cells expressing PD-L1 at IHC) non-ACC were: carcinoma ex pleomorphic adenoma (36\%), salivary duct carcinoma (30\%), large cell carcinoma (10\%), adenocarcinoma NOS (8\%) [77]. Moreover, higher PD-L1 expression was associated with a shorter disease-free survival (hazard ratio for recurrence $2.28, p=0.008$ ).

Among 26 salivary gland cancer patients (all PD-L1 positive) treated with anti-PD-1 (pembrolizumab) within a phase 1b clinical trial, $11.5 \%$ of major responses ( 3 salivary gland tumors: 2 adenocarcinoma, 1 high grade serous carcinoma) were observed, with a 17-weeks median duration of response [78]. Experimental trials are ongoing to investigate the role of $\mathrm{IO}$ in salivary gland carcinomas, either with anti-PD-1 as single agent [79] or anti-PD-1 and anti-CTLA4 as immune combination [80]. The identification of predictive biomarkers is an unmet clinical need over all cancer types.

\subsection{Gene fusions}

In addition to the aforementioned genomic alterations, salivary gland malignancies can be driven by recurrent gene fusions as well. Even though many of them are not druggable, the presence of a specific translocation could be pathognomonic for a particular subtypes [81] [82]. Other recurrent genetic fusions are CRTC1-MAML2 in mucoepidermoid carcinoma, especially in low/intermediate grade types [83], and MECT1-MAML2 translocation, which can be found in up to $88 \%$ cases of mucoepidermoid carcinoma [84]. EWRS1-ATF1 was reported in cases of hyalinizing clear cell salivary gland carcinoma [85]. Acinic cell carcinoma might bear HTN3-MSANTD3 translocation, leading to upregulation of genes involved in regulation of translation [86]. 
Myoepithelial carcinoma were found to be related either to FGFR-PLAG1 or PLAG1-TGFR $\beta$, respectively in myoepithelial carcinoma ex pleomorphic adenoma and in myoepithelial carcinoma de novo. These findings suggest a different genesis and prognosis in these two groups (good prognosis in PLAG1-TGFR $\beta$ translocated de novo myoepithelial carcinoma while poor prognosis in myoepithelial carcinoma with high copy number alterations arising from FGFR1-PLAG1 translocated ones) [55].

Moreover, huge clinical responses with a RET inhibitor (cabozantinib) have been already reported in salivary duct carcinoma and adenocarcinoma NOS [40]. This could be an interesting hypothesis-generating finding justifying the possibility to explore the role of RET inhibitors (e.g. cabozantinib, LOXO-292 and BLU-667 for medullary thyroid and non-small cell lung cancer [87] [88]) in RET mutated or rearranged tumors. Clinical trials [89] are ongoing in this setting.

\section{Conclusions}

Genomic alterations should be investigated in specific histotypes of non-ACC salivary gland malignancies. In this cancer patient population, improving the knowledge of the biological background could help clinicians tailor treatments and focus their research perspectives. Further investigations are necessary to explore the role of molecular-driven targeted agents in the curative setting.

\section{Expert Opinion}

Our review is born from an important unmet clinical need such as to find effective systemic therapies in the group of salivary gland malignant tumors other than ACC. To date, the lack of active treatments as well as clinical trials as alternative to chemotherapy frequently may lead the oncologists to enroll patients in phase I trials. This is appreciated, and it should be still encouraged but this practice, without a very solid biological rationale, could lead patients, their families and physicians to an unnecessary waste of energy and costs. Recently, a new way to give therapeutic indications in medical oncology is emerging and it could find practical application in this context: a biomarker-selected strategy, regardless of any cancer site. It is an agnostic way to prescribe therapies which has already been applied by Food and Drug Administration (FDA) in cancer patients and it is even more appreciated in the context of rare cancers where the therapeutic opportunities are scant in most of cases. In fact, pembrolizumab (anti-PD-1 agent) was FDAapproved for advanced solid tumors with high MSI or deficiency of Mismatch Repair (d-MMR) system while larotrectinib for [48] those tumors with NTRK gene fusions [90].

In this overview, we summarize the most relevant druggable molecular alterations in non-ACC. For recurrent or metastatic non-ACC patients, the genetic profiling by in-depth molecular analyses (e.g. NGS) of the tumor tissue is strongly advised to identify any potentially druggable target. Noteworthy, non-ACC group includes more than twenty histotypes completely different each other reflecting a potential diverse genetic background. Moreover, high-grade poorly-differentiated salivary gland carcinomas harbored higher number of gene alterations compared to low- or intermediate grade ones [39] suggesting that each histotype may correspond to more disease entities.

On one hand, the genetics-based approach could overcome a conventional issue of these tumors: difficulties in designing and conducting trials due to small numbers of patients when divided according to histology. 
On the other hand, this new approach has also many limitations. First, the techniques used to detect gene alterations (e.g. NGS) are not still homogeneous in terms of type and/or number of identifiable mutations: a harmonization process is strictly recommended. Second, the financial burden of NGS-oriented approach is consistent and not sustainable by all international healthcare providers. Third, this method will not be able to uncover all effective therapies in non-ACC. In fact, these tumors have a low rate of gene alterations and their landscape may be characterized by further steps of regulation (e.g. epigenomics etc) and intratumor heterogeneity thus unabling to effectively targeting DNA alterations.

After all, a more extensive use of genomic profiling could fill the gaps of the available series whose main limitations are: 1) the lack of correspondence with clinical history, outcomes, performed therapies and all other clinical data; 2) the samples used for genomic profiling were often archival tissues, not so representative of advanced disease where genetic changes from baseline situation may be appeared.

Bearing this in mind, a greatly conceptual and practical cooperation of pharma companies, academy and cancer referral centers is advocated. Patient representatives and their support groups should be also fully involved in this process. The recent activation of such networks, as the European Reference Networks (ERN), is a significant chance for clinicians to share experiences and knowledge [91]. In the meantime, referral to highly specialized hospitals is strongly recommended to optimize the management of these patients. In particular, histopathologic diagnosis should be always reviewed by expert pathologists, whose skills have to be progressively updated.

In this setting, the dissemination of this practice and the clinical/preclinical research should be implemented throughout all these networks. Furthermore, salivary gland cancer patients and their families should be accurately informed regarding all possible ethical implications of molecular analyses, also for patients' relatives. These points are just a few suggestions aimed both at improving the counseling and supporting a more strongly evidence-based physician-patient relationship, in the era of personalized medicine, which is also participatory.

\section{Funding}

This paper was not funded

\section{Declaration of interest}

L Licitra has disclosed funding (to her institution) for clinical studies and research from AstraZeneca, Boehringer Ingelheim, Eisai, Merck Serono, MSD, Novartis, and Roche, has received compensation for service as a consultant/advisor and/or for lectures from AstraZeneca, Bayer, Bristol-Myers Squibb, Boehringer Ingelheim, Debiopharm, Eisai, Merck Serono, MSD, Novartis, Roche, and Sobi; and has received travel coverage for meetings from Bayer, Bristol-Myers Squibb, Debiopharm, Merck Serono, MSD, and Sobi. P Bossi has disclosed honoraria or consultation fees from Roche, Merck Serono, Mundipharma, Kyowa Kirin, AstraZeneca. LD Locati has disclosed honoraria or consultation fees from Eisai. The authors have no other relevant affiliations or financial involvement with any organization or entity with a financial interest in or financial conflict with the subject matter or materials discussed in the manuscript. This includes employment, consultancies, honoraria, stock ownership or options, expert testimony, grants or patents received or pending, or royalties. 


\section{$\underline{\text { Reviewer disclosures }}$}

Peer reviewers on this manuscript have no relevant financial or other relationships to disclose

\section{References}

Papers of special note have been highlighted as either of interest $(\bullet)$ or of considerable interest $(\bullet \bullet)$ to readers

1. WHO, WHO Classification of Head and Neck Tumours - 4th Edition, ed. A.E.-N.J.C.J.G.T.T.P. Slootweg. 2017.

** Fundamental reference for clinicians and pathologists dealing with salivary gland cancers

2. Laurie, S.A. and L. Licitra, Systemic therapy in the palliative management of advanced salivary gland cancers. J Clin Oncol, 2006. 24(17): p. 2673-8.

3. Ho, A.S., et al., The mutational landscape of adenoid cystic carcinoma. Nat Genet, 2013. 45(7): p. 791-8.

* Review of the mutational landscape of adenoid cystic carcinoma

4. Ross, J.S., et al., Comprehensive genomic profiling of relapsed and metastatic adenoid cystic carcinomas by next-generation sequencing reveals potential new routes to targeted therapies. Am J Surg Pathol, 2014. 38(2): p. 235-8.

5. Yin, L.X. and P.K. Ha, Genetic alterations in salivary gland cancers. Cancer, 2016. 122(12): p. 182231.

6. Grunewald, I., et al., Targeted next generation sequencing of parotid gland cancer uncovers genetic heterogeneity. Oncotarget, 2015. 6(20): p. 18224-37.

7. Kato, S., et al., Genomic landscape of salivary gland tumors. Oncotarget, 2015. 6(28): p. 25631-45.

* Review of the mutational landscape of salivary gland malignancies

8. Ross, J.S., et al., Comprehensive genomic profiles of metastatic and relapsed salivary gland carcinomas are associated with tumor type and reveal new routes to targeted therapies. Ann Oncol, 2017. 28(10): p. 2539-2546.

** Outstanding report of a wide assessment of genomic profiling in advanced salivary gland cancers

9. Alexandrov, L.B., et al., Signatures of mutational processes in human cancer. Nature, 2013. 500(7463): p. 415-21.

10. Petitjean, A., et al., TP53 mutations in human cancers: functional selection and impact on cancer prognosis and outcomes. Oncogene, 2007. 26(15): p. 2157-65.

11. Locati, L.D., et al., Treatment relevant target immunophenotyping of 139 salivary gland carcinomas (SGCS). Oral Oncol, 2009. 45(11): p. 986-90.

* Report of a large single center experience of salivary drug cancers analyzed through immunohistochemistry to detect druggable targets

12. Mitani, Y., et al., Alterations associated with androgen receptor gene activation in salivary duct carcinoma of both sexes: potential therapeutic ramifications. Clin Cancer Res, 2014. 20(24): p. 657081.

13. Gilbert, M.R., et al., A 20-Year Review of 75 Cases of Salivary Duct Carcinoma. JAMA Otolaryngol Head Neck Surg, 2016. 142(5): p. 489-95.

14. Dalin, M.G., et al., Androgen Receptor Signaling in Salivary Gland Cancer. Cancers (Basel), 2017. 9(2).

15. Locati, L.D., et al., Clinical activity of androgen deprivation therapy in patients with metastatic/relapsed androgen receptor-positive salivary gland cancers. Head Neck, 2016. 38(5): p. 724-31. 
16. Dalin, M.G., et al., Comprehensive Molecular Characterization of Salivary Duct Carcinoma Reveals Actionable Targets and Similarity to Apocrine Breast Cancer. Clin Cancer Res, 2016. 22(18): p. 462333.

17. Alfieri, S., et al., Systemic therapy in metastatic salivary gland carcinomas: A pathology-driven paradigm? Oral Oncol, 2017. 66: p. 58-63.

* Review of systemic treatments for salivary gland cancers

18. Fushimi, C., et al., A prospective phase II study of combined androgen blockade in patients with androgen receptor-positive metastatic or locally advanced unresectable salivary gland carcinoma. Ann Oncol, 2018. 29(4): p. 979-984.

19. Boon, E., et al., Androgen deprivation therapy for androgen receptor-positive advanced salivary duct carcinoma: A nationwide case series of 35 patients in The Netherlands. Head Neck, 2018. 40(3): p. 605-613.

20. Locati, L.D., et al., Activity of abiraterone in rechallenging two AR-expressing salivary gland adenocarcinomas, resistant to androgen-deprivation therapy. Cancer Biol Ther, 2014. 15(6): p. 67882.

21. Enzalutamide for Patients With Androgen Receptor Positive Salivary Cancers - NCT02749903. 07Dec-2018]; Available from: https://clinicaltrials.gov/ct2/show/NCT02749903.

22. Cappelletti, V., et al., Tailoring treatment of salivary duct carcinoma (SDC) by liquid biopsy: ARv7 expression in circulating tumor cells. Ann Oncol, 2018. 29(7): p. 1598-1600.

23. Udager, A.M. and S.I. Chiosea, Salivary Duct Carcinoma: An Update on Morphologic Mimics and Diagnostic Use of Androgen Receptor Immunohistochemistry. Head Neck Pathol, 2017. 11(3): p. 288-294.

24. Locati, L.D., et al., Cetuximab in recurrent and/or metastatic salivary gland carcinomas: A phase II study. Oral Oncol, 2009. 45(7): p. 574-8.

25. Jakob, J.A., et al., Phase II study of gefitinib in patients with advanced salivary gland cancers. Head Neck, 2015. 37(5): p. 644-9.

26. Cros, J., et al., Expression and mutational status of treatment-relevant targets and key oncogenes in 123 malignant salivary gland tumours. Ann Oncol, 2013. 24(10): p. 2624-9.

27. Dagrada, G.P., et al., Expression of HER-2/neu gene and protein in salivary duct carcinomas of parotid gland as revealed by fluorescence in-situ hybridization and immunohistochemistry. Histopathology, 2004. 44(3): p. 301-2.

28. Clauditz, T.S., et al., Human epidermal growth factor receptor 2 (HER2) in salivary gland carcinomas. Pathology, 2011. 43(5): p. 459-64.

29. Ettl, T., et al., LoSS of PTEN is associated with elevated EGFR and HER2 expression and worse prognosis in salivary gland cancer. Br J Cancer, 2012. 106(4): p. 719-26.

30. Ettl, T., et al., EGFR, HER2, survivin, and loss of PSTAT3 characterize high-grade malignancy in salivary gland cancer with impact on prognosis. Hum Pathol, 2012. 43(6): p. 921-31.

31. Andreasen, S., M. Grauslund, and S. Heegaard, Lacrimal gland ductal carcinomas: Clinical, Morphological and Genetic characterization and implications for targeted treatment. Acta Ophthalmol, 2017. 95(3): p. 299-306.

32. Haddad, R., et al., Herceptin in patients with advanced or metastatic salivary gland carcinomas. A phase II study. Oral Oncol, 2003. 39(7): p. 724-7.

33. Limaye, S.A., et al., Trastuzumab for the treatment of salivary duct carcinoma. Oncologist, 2013. 18(3): p. 294-300.

34. Agulnik, M., et al., Phase II study of lapatinib in recurrent or metastatic epidermal growth factor receptor and/or erbB2 expressing adenoid cystic carcinoma and non adenoid cystic carcinoma malignant tumors of the salivary glands. J Clin Oncol, 2007. 25(25): p. 3978-84.

35. My Pathway: A Study Evaluating Herceptin/Perjeta, Tarceva, Zelboraf/Cotellic, Erivedge, Alecensa, and Tecentriq Treatment Targeted Against Certain Molecular Alterations in Participants With Advanced Solid Tumors - NCT02091141.

36. van Boxtel, W., et al., Combination of docetaxel, trastuzumab and pertuzumab or treatment with trastuzumab-emtansine for metastatic salivary duct carcinoma. Oral Oncol, 2017. 72: p. 198-200. 
37. Takahashi, H., et al., Phase II Trial of Trastuzumab and Docetaxel in Patients With Human Epidermal Growth Factor Receptor 2-Positive Salivary Duct Carcinoma. J Clin Oncol, 2018: p. JCO1800545.

38. Can, N.T., et al., Expression of Hormone Receptors and HER-2 in Benign and Malignant Salivary Gland Tumors. Head Neck Pathol, 2018. 12(1): p. 95-104.

39. Wang, K., et al., Comprehensive genomic profiling of salivary mucoepidermoid carcinomas reveals frequent BAP1, PIK3CA, and other actionable genomic alterations. Ann Oncol, 2017. 28(4): p. 748753.

40. Wang, K., et al., Profiling of 149 Salivary Duct Carcinomas, Carcinoma Ex Pleomorphic Adenomas, and Adenocarcinomas, Not Otherwise Specified Reveals Actionable Genomic Alterations. Clin Cancer Res, 2016. 22(24): p. 6061-6068.

41. Clauditz, T.S., et al., Abundant expression of mTOR kinase in salivary gland tumors - potentials as therapy target? J Oral Pathol Med, 2013. 42(10): p. 769-73.

42. Skalova, A., et al., Mammary analogue secretory carcinoma of salivary glands, containing the ETV6NTRK3 fusion gene: a hitherto undescribed salivary gland tumor entity. Am J Surg Pathol, 2010. 34(5): p. 599-608.

43. Skalova, A., et al., Molecular Profiling of Mammary Analog Secretory Carcinoma Revealed a Subset of Tumors Harboring a Novel ETV6-RET Translocation: Report of 10 Cases. Am J Surg Pathol, 2018. 42(2): p. 234-246.

44. Andreasen, S., et al., ETV6 Gene Rearrangements Characterize a Morphologically Distinct Subset of Sinonasal Low-grade Non-intestinal-type Adenocarcinoma: A Novel Translocation-associated Carcinoma Restricted to the Sinonasal Tract. Am J Surg Pathol, 2017. 41(11): p. 1552-1560.

45. Andreasen, S., et al., The ETV6-RET Gene Fusion Is Found in ETV6-rearranged Low-grade Sinonasal Adenocarcinoma Without NTRK3 Involvement. Am J Surg Pathol, 2018. 42(7): p. 985-988.

46. Menichincheri, M., et al., Discovery of Entrectinib: A New 3-Aminoindazole As a Potent Anaplastic Lymphoma Kinase (ALK), c-ros Oncogene 1 Kinase (ROS1), and Pan-Tropomyosin Receptor Kinases (Pan-TRKs) inhibitor. J Med Chem, 2016. 59(7): p. 3392-408.

47. Drilon, A., et al., Efficacy of Larotrectinib in TRK Fusion-Positive Cancers in Adults and Children. N Engl J Med, 2018. 378(8): p. 731-739.

48. Drilon, A., et al., What hides behind the MASC: clinical response and acquired resistance to entrectinib after ETV6-NTRK3 identification in a mammary analogue secretory carcinoma (MASC). Ann Oncol, 2016. 27(5): p. 920-6.

49. Demetri, G.D.P.-A., L.; Farago, A.F., LBA17 - Efficacy and Safety of Entrectinib in Patients with NTRK Fusion-Positive (NTRK-fp) Tumors: Pooled Analysis of STARTRK-2, STARTRK-1 and ALKA-372-001. 2018.

50. Finn, R.S., et al., Palbociclib and Letrozole in Advanced Breast Cancer. N Engl J Med, 2016. 375(20): p. 1925-1936.

51. Goetz, M.P., et al., MONARCH 3: Abemaciclib As Initial Therapy for Advanced Breast Cancer. J Clin Oncol, 2017. 35(32): p. 3638-3646.

52. Turner, N.C., et al., Overall Survival with Palbociclib and Fulvestrant in Advanced Breast Cancer. N Engl J Med, 2018. 379(20): p. 1926-1936.

53. Porta, R., et al., FGFR a promising druggable target in cancer: Molecular biology and new drugs. Crit Rev Oncol Hematol, 2017. 113: p. 256-267.

54. Ach, T., et al., Genomic aberrations of MDM2, MDM4, FGFR1 and FGFR3 are associated with poor outcome in patients with salivary gland cancer. J Oral Pathol Med, 2016. 45(7): p. 500-9.

55. Dalin, M.G., et al., Multi-dimensional genomic analysis of myoepithelial carcinoma identifies prevalent oncogenic gene fusions. Nat Commun, 2017. 8(1): p. 1197.

56. Lord, C.J. and A. Ashworth, PARP inhibitors: Synthetic lethality in the clinic. Science, 2017. 355(6330): p. 1152-1158.

57. Martens-de Kemp, S.R., et al., The FA/BRCA Pathway Identified as the Major Predictor of Cisplatin Response in Head and Neck Cancer by Functional Genomics. Mol Cancer Ther, 2017. 16(3): p. 540550.

58. Nardi, V., et al., Detection of novel actionable genetic changes in salivary duct carcinoma helps direct patient treatment. Clin Cancer Res, 2013. 19(2): p. 480-90. 
59. Ho, A.L., Preliminary results from a phase 2 trial of tipifarnib in Squamous Cell Carcinomas (SCCS) with HRAS mutations. Annals of Oncology, 2018. 29 (suppl 8): p. viii372-viii399.

60. Choudhry, Z., et al., Sonic hedgehog signalling pathway: a complex network. Ann Neurosci, 2014. 21(1): p. 28-31.

61. Gupta, S., N. Takebe, and P. Lorusso, Targeting the Hedgehog pathway in cancer. Ther Adv Med Oncol, 2010. 2(4): p. 237-50.

62. Vidal, M.T., et al., The sonic hedgehog signaling pathway contributes to the development of salivary gland neoplasms regardless of perineural infiltration. Tumour Biol, 2016. 37(7): p. 9587-601.

63. Sekulic, A., et al., Efficacy and safety of vismodegib in advanced basal-cell carcinoma. N Engl J Med, 2012. 366(23): p. 2171-9.

64. Rodon, J., et al., A phase I, multicenter, open-label, first-in-human, dose-escalation study of the oral smoothened inhibitor Sonidegib (LDE225) in patients with advanced solid tumors. Clin Cancer Res, 2014. 20(7): p. 1900-9.

65. Gelsomino, F., et al., Targeting the MET gene for the treatment of non-small-cell lung cancer. Crit Rev Oncol Hematol, 2014. 89(2): p. 284-99.

66. Ma, P.C., et al., Expression and mutational analysis of MET in human solid cancers. Genes Chromosomes Cancer, 2008. 47(12): p. 1025-37.

67. Garcia-Vilas, J.A. and M.A. Medina, Updates on the hepatocyte growth factor/c-Met axis in hepatocellular carcinoma and its therapeutic implications. World J Gastroenterol, 2018. 24(33): p. 3695-3708.

68. Ach, T., et al., Aberrations of MET are associated with copy number gain of EGFR and loss of PTEN and predict poor outcome in patients with salivary gland cancer. Virchows Arch, 2013. 462(1): p. 6572.

69. Snyder, A., et al., Genetic basis for clinical response to CTLA-4 blockade in melanoma. N Engl J Med, 2014. 371(23): p. 2189-2199.

70. Rizvi, N.A., et al., Cancer immunology. Mutational landscape determines sensitivity to PD-1 blockade in non-small cell lung cancer. Science, 2015. 348(6230): p. 124-8.

71. Goodman, A.M., et al., Tumor Mutational Burden as an Independent Predictor of Response to Immunotherapy in Diverse Cancers. Mol Cancer Ther, 2017. 16(11): p. 2598-2608.

72. Goto, Y., Tumor Mutation Burden: Is It Ready for the Clinic? J Clin Oncol, 2018: p. JCO2018793398.

73. Ishida, Y., et al., Induced expression of PD-1, a novel member of the immunoglobulin gene superfamily, upon programmed cell death. EMBO J, 1992. 11(11): p. 3887-95.

74. Francisco, L.M., P.T. Sage, and A.H. Sharpe, The PD-1 pathway in tolerance and autoimmunity. Immunol Rev, 2010. 236: p. 219-42.

75. Hodi, F.S., et al., Nivolumab plus ipilimumab or nivolumab alone versus ipilimumab alone in advanced melanoma (CheckMate 067): 4-year outcomes of a multicentre, randomised, phase 3 trial. Lancet Oncol, 2018. 19(11): p. 1480-1492.

76. Reck, M., et al., Pembrolizumab versus Chemotherapy for PD-L1-Positive Non-Small-Cell Lung Cancer. N EnglJ Med, 2016. 375(19): p. 1823-1833.

77. Mukaigawa, T., et al., Programmed death ligand-1 expression is associated with poor disease free survival in salivary gland carcinomas. J Surg Oncol, 2016. 114(1): p. 36-43.

78. Cohen, R.B., Preliminary results for the advanced salivary gland carcinoma cohort of the phase $1 b$ KEYNOTE-028 study of pembrolizumab. . J Clin Oncol 2016. 34 (suppl): p. 6017.

79. Nivolumab in Recurrent or Metastatic Salivary Gland Carcinoma of the Head and Neck (NISCAHN) NCT03132038. 07-Dec-2018]; Available from: https://clinicaltrials.gov/ct2/show/NCT03132038.

80. Nivolumab and Ipilimumab in Treating Patients With Metastatic/Recurrent ACC of All Sites and NonACC Salivary Gland Cancer - NCT03146650. 07-Dec-2018]; Available from: https://clinicaltrials.gov/ct2/show/NCT03146650.

81. Stenman, G., Fusion oncogenes in salivary gland tumors: molecular and clinical consequences. Head Neck Pathol, 2013. 7 Suppl 1: p. S12-9.

82. Weinreb, I., Translocation-associated salivary gland tumors: a review and update. Adv Anat Pathol, 2013. 20(6): p. 367-77. 
83. Tirado, Y., et al., CRTC1/MAML2 fusion transcript in high grade mucoepidermoid carcinomas of salivary and thyroid glands and Warthin's tumors: implications for histogenesis and biologic behavior. Genes Chromosomes Cancer, 2007. 46(7): p. 708-15.

84. Kang, H., et al., Whole-Exome Sequencing of Salivary Gland Mucoepidermoid Carcinoma. Clin Cancer Res, 2017. 23(1): p. 283-288.

85. Nakano, T., et al., Hyalinizing clear cell carcinoma with EWSR1-ATF1 fusion gene: report of three cases with molecular analyses. Virchows Arch, 2015. 466(1): p. 37-43.

86. Andreasen, S., et al., The HTN3-MSANTD3 Fusion Gene Defines a Subset of Acinic Cell Carcinoma of the Salivary Gland. Am J Surg Pathol, 2018.

87. Subbiah, V., et al., Selective RET kinase inhibition for patients with RET-altered cancers. Ann Oncol, 2018. 29(8): p. 1869-1876.

88. Subbiah, V., et al., Precision Targeted Therapy with BLU-667 for RET-Driven Cancers. Cancer Discov, 2018. 8(7): p. 836-849.

89. Cabozantinib in Advanced Salivary Gland Cancer Patients (Cabo ASAP) - NCT03729297. 07-Dec2018]; Available from: https://clinicaltrials.gov/ct2/show/NCT03729297.

90. FDA approves larotrectinib for solid tumors with NTRK gene fusions. 07-Dec-2018]; Available from: https://www.fda.gov/Drugs/InformationOnDrugs/ApprovedDrugs/ucm626720.htm.

91. Frezza, A.M., et al., Networking in rare cancers: What was done, what's next. Eur J Surg Oncol, 2018.

* Review about the importance and the opportunities of networking in rare malignancies 


\begin{tabular}{|l|}
\hline Histotype \\
\hline Mucoepidermoid carcinoma (MEC) \\
\hline Acinic cell carcinoma \\
\hline Polymorphous adenocarcinoma \\
\hline Clear cell carcinoma \\
\hline Basal cell carcinoma (BCC) \\
\hline Intraductal carcinoma (IDC) \\
\hline Adenocarcinoma not otherwise specified (ADC NOS) \\
\hline Salivary duct carcinoma (SDC) \\
\hline Myoepithelial carcinoma \\
\hline Epithelial-myoepithelial carcinoma \\
\hline Carcinoma ex pleomorphic adenoma \\
\hline Secretory carcinoma \\
\hline Sebaceous adenocarcinoma \\
\hline Carcinosarcoma \\
\hline Soorly differentiated carcinoma \\
\hline Oncocytic carcinoma \\
\hline
\end{tabular}

Table 1 - Malignant epithelial salivary gland tumors, non adenoid-cystic carcinoma histotypes [1] 
table_2

\begin{tabular}{|c|c|c|c|c|c|c|c|c|}
\hline Druggable molecular target & AcCC & ADC NOS & Ca ex pleom. ad & MASC & MEC & MyoepiC & PLGAC & SDC \\
\hline PI3K & & $24 \%$ & & & $4.3 \%$ & $15 \%$ & & $27 \%$ \\
\hline TRK & & & & $100 \%$ & & & & \\
\hline CDK / Cyclin & $71 \%$ & $35 \%$ & & & $0 \% \quad(A G)$ & $33 \%$ & & \\
\hline FGFR & & & $9 \%$ & & $(A G)$ & & $20 \%$ & \\
\hline BRCA & & & & & $10.5 \% \quad(\mathrm{AG})$ & & & \\
\hline RAS / RAF / MAPK & $5 \%$ & $5 \%$ & & & & & & $5 \%$ \\
\hline Hedgehog & & & & & & $10 \%$ & & \\
\hline MET & & CR & & & & CR & & \\
\hline High* TMB & & $10 \%$ & $12 \%$ & & $10 \%$ & & & $14 \%$ \\
\hline PD1 / PD-L1 & & $8 \%$ & $36 \%$ & & & & & $30 \%$ \\
\hline
\end{tabular}

Table 1 - Overview of associations between potentially druggable molecular targets and non-ACC SGC histotype $[7,8,17]$

* defined as > 10 mut/MB;

Abbreviations: non-ACC, non adenoid cystic carcinoma; HER-2, human epidermal growth factor type 2; TMB, tumor mutational burden; AcCC, acinic cell carcinoma; ADC NOS, adenocarcinoma not otherwise specified; Ca ex pleom. ad, carcinoma ex pleomorphic adenoma; MASC, mammary analogue secretory 
carcinoma; MEC, mucoepidermoid carcinoma; MyoepiC, myoepithelial carcinoma; PLGAC, pleomorphic low grade adenocarcinoma; SDC, salivary duct carcinoma; HG, high grade; LIG, low-intermediate grade; AG, all grades;

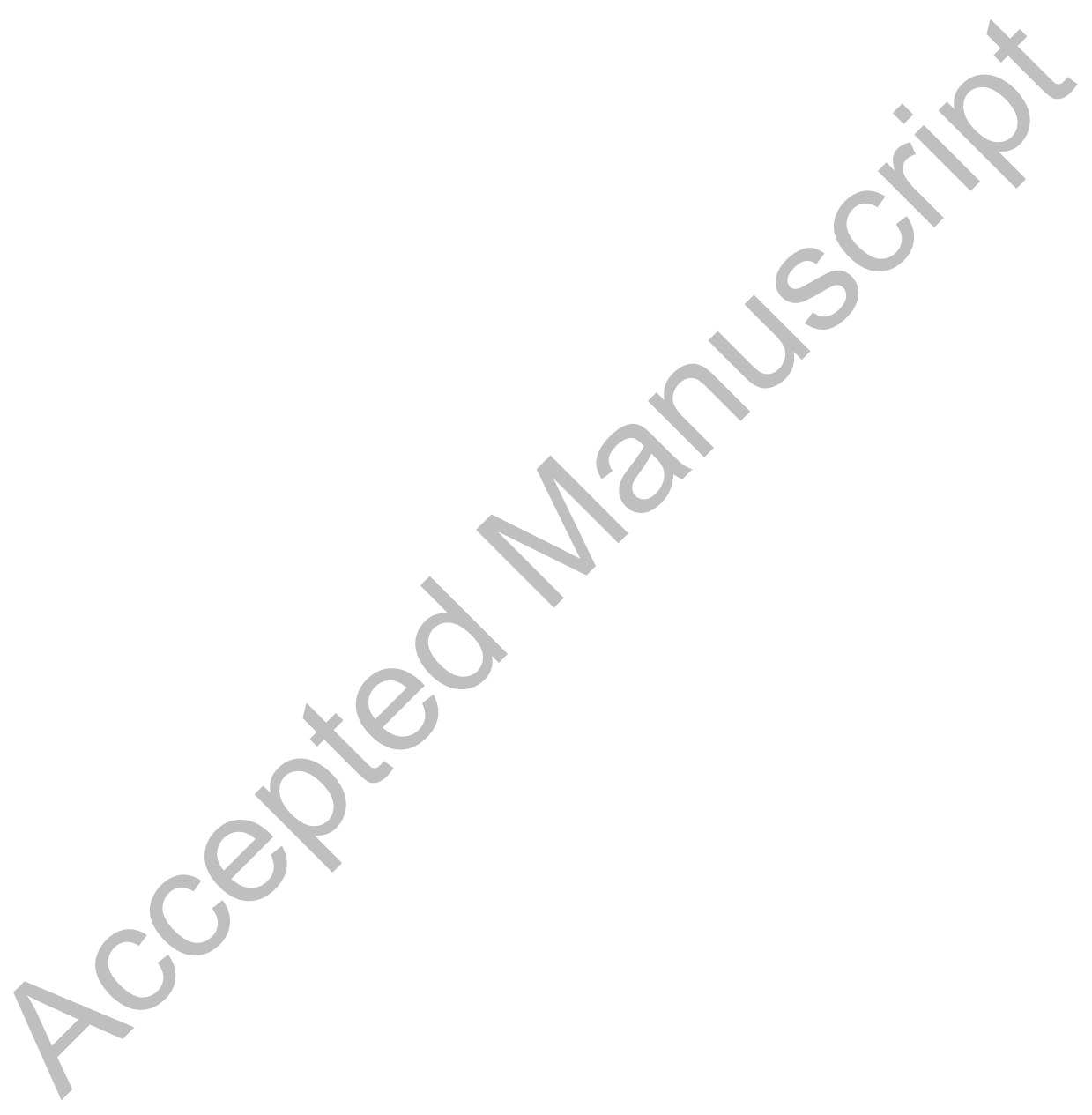

